\title{
Variability in Microleakage Observed in a Total-etch Wet-bonding Technique under Different Handling Conditions
}

\author{
F.R. Tay, A.J. Gwinnett², K.M. Pang1, and S.H.Y. Wei
}

Department of Children's Dentistry and Orthodontics, ${ }^{1}$ Oral Biology Unit, Faculty of Dentistry, The University of Hong Kong, 34 Hospital Road, Hong Kong; '2Department of Oral Biology and Pathology, School of Dental Medicine, State University of New York at Stony Brook, New York 11794-8702, USA; " to whom correspondence should be addressed, Dean's Office, Prince Philip Dental Hospital, 34, Hospital Road, Pokfulam, Hong Kong

\begin{abstract}
Using a stereoscopic clearing protocol and scanning electron microscopy, we investigated the extent and nature of microleakage in a total-etch, currentgeneration dentin adhesive by a wet-bonding technique under different handling conditions. The hypotheses were that inadequate light curing of the primer or incomplete drying of the primer solvent might adversely affect the sealing ability of an acetone-containing adhesive system. The study consisted of three experimental groups: (I) a control group with an adequate light source and with the primer solvent completely dried; (II) an "inadequate-light" group; and (III) an "incomplete evaporation of primer solvent" group. The extent of microleakage after silver staining and clearing of the specimens was scored based on a modified five-point scale. Nonparametric statistical analysis (Kruskal-Wallis ANOVA) followed by a multiple comparison test (Dunn test) indicated significant differences among the three groups $(\mathrm{p}<0.05)$.

SEM examination of the restorative interface revealed that microleakage appeared to be initiated from the bonding resin-hybrid layer interface in all three groups, representing the weak link in the adhesive system. In addition, microleakage was characterized by 5 zones, each delineating a stage in a continuous array of progressively deleterious microleakage patterns variously distributed among the three groups. It was suggested that, while the bonding resin-hybrid layer interface represented the intrinsic weakness in an already much improved dentin adhesive, extrinsic factors such as the adequacy of the curing light and, more importantly, complete removal of the primer solvents can and should be avoided to preserve the structural integrity of the marginal seal.
\end{abstract}

Key words: microleakage, weak link, resin-dentin interface, clearing, SEM.

Received June 17, 1994; Accepted December 30, 1994

\section{Introduction}

The quality and durability of a marginal seal in dentin devoid of enamel have always been among the prime concerns affecting the clinician's choice of a dentin adhesive system. With previous generations of dentin bonding agents, inadequate marginal adaptation and postrestorative gap formation (Davila et al., 1988; Suzuki et al., 1989) have been attributed to polymerization contraction of light-cured resin composites (Feilzer et al., 1988; Fusayama, 1992a), with important clinical consequences (Triadan, 1987; Cox, 1992). For some of the current adhesive systems, the use of the total-etch technique (Bertolotti, 1990; Fusayama, $1992 b)$, together with the application of acetone- or alcoholcontaining primer resins on a wet dentin substrate (Gwinnett, 1992), has resulted in gap-free restorations in vivo (Gwinnett and Kanca, 1992a; Gwinnett et al., 1992). The structural evidence of such a sealed tissue interface in vivo has been elucidated (Tay et al., 1994a).

Despite these potentially encouraging results, there have been instances of persistent post-operative sensitivity following total etching and the application of certain dentin adhesives, in particular those containing acetone. Dentin hypersensitivity, a manifestation of the sensory component of microleakage (Cox, 1992), results from hydrodynamic fluid movement within the dentinal tubule complex (Brännström et al., 1967). This clinical phenomenon can occur despite careful isolation prior to direct restorative procedures and the use of an incremental filling technique, as well as after cementation of prefabricated restorations in conjunction with resin cements.

Based on our preliminary bond strength and scanning electron microscopic (SEM) fractographic studies, we hypothesized that such a clinical phenomenon might be related to : (a) the use of an inadequate light-curing source, or (b) incomplete evaporation of the primer solvents prior to application of the bonding resin. The aim of this study was to test the hypotheses that such operator-controllable variables have an adverse effect on the sealing ability of an 
acetone-containing dentin adhesive system, manifested as an increase in the severity of microleakage.

Traditional in vitro microleakage assessment of the extent of dye/tracer penetration in sectioned specimens raised the problem of interpreting a three-dimensional event on a twodimensional level (Taylor and Lynch, 1992). Furthermore, information on the exact interfacial location of microleakage is sparse, and micrographs of such leakage interfaces are usually taken at low magnification. It has also been estimated that traditional assessment methods did not detect the exact location of microleakage in $75 \%$ of the cases where current dentin adhesives were assessed (Garcia-Godoy and Finger, 1993). We anticipated that a study of the extent and nature of microleakage would provide insight into a possible cause-and-effect relationship of this clinical problem. The present study investigated the microleakage patterns in a total-etch wet-bonding technique when the AllBond 2 system (Bisco Dental Products, Itasca, IL) was handled under the two previously hypothesized variables. Using a stereoscopic clearing protocol, we assessed the extent of microleakage. We used secondary (SEI) and backscattered (BSE) scanning electron microscopy to assess the exact pathway of microleakage.

\section{Materials and methods}

Forty-five freshly extracted, intact, caries-free human third molars were hand-scaled, cleaned with a slurry of pumice, stored in $0.5 \%$ chloramine $\mathrm{T}$ at $5^{\circ} \mathrm{C}$, and used within one month of extraction. A cylindrical cavity preparation was placed at the cemento-enamel junction (CEJ) of the buccal surface of each tooth with half of the restoration above the CEJ and the remainder below the CEJ. The cavities were prepared with No. 170 carbide burs in a high-speed handpiece equipped with water spray. The preparations were made as uniformly as possible (approximately $4 \mathrm{~mm}$ in diameter and $2 \mathrm{~mm}$ deep) with a short enamel bevel. The gingival margin, which was located in dentin/cementum, was not beveled.

Two dental curing units (Max Lite, L.D. Caulk/Dentsply, Milford, DE) of different light intensities were used in the experiment. Their outputs were registered with a light meter (Cure Rite, L.D. Caulk/Dentsply, Milford, DE). The first unit, with a new filter and a new $150 \mathrm{~W}$ light bulb, registering an output of $420 \mathrm{~mW} / \mathrm{cm}^{2}$, was designated as the adequate light. The second, with an output of $68 \mathrm{~mW} / \mathrm{cm}^{2}$, was designated as the inadequate light. The same tip size (diameter, $10 \mathrm{~mm}$ ) was used for both the adequate and inadequate lights.

The specimens were divided randomly into three treatment groups of 15 teeth each. We restored them all with a total-etch, wet-bonding technique, using the All-Bond 2 system with variations in the handling of the All-Bond 2 primers in each category.

\section{Control}

The enamel and dentin were conditioned with $10 \%$ phosphoric acid gel etchant (All Etch semigel, Bisco, Itasca, IL) for $20 \mathrm{sec}$ and rinsed for $20 \mathrm{sec}$. A wet-bonding technique was used as recommended by the manufacturer. Since there were no solid guidelines in the manufacturer's instructions regarding how moist is "moist dentin", we adopted a standardized protocol throughout the experiment by gently blow-drying the conditioned preparation with oil/dust-free air (Dust-Off Plus, Falcon Safety Products, Inc., Branchburg, NJ) for approximately three sec until there were no visible signs of water on the dentin surface. One drop each of primer A, containing N-ptolylglycine-glycidyl methacrylate (NTG-GMA), and primer B, containing biphenyl dimethacrylate (BPDM), was dispensed from the bonding kit and mixed in a well. The thoroughly mixed reagent was applied by multiple brush applications (5 coats) to achieve a shiny film of resin on the substrate. The primer-infiltrated substrate surface was gently air-dried for 10 sec so that the primer solvent could evaporate completely. This was followed by light curing the primed surface with the adequate light $\left(420 \mathrm{~mW} / \mathrm{cm}^{2}\right)$ for $20 \mathrm{sec}$. A thin layer of dentinenamel bonding resin was then applied to the primed dentin surface, air-thinned, and light-polymerized for $20 \mathrm{sec}$. The rest of the cavity was incrementally restored with a hybrid resin composite (Z100-A3 shade, 3M Co., St. Paul, MN) in increments of $1 \mathrm{~mm}$ and light-activated for $40 \mathrm{sec}$. Excessive materials were removed after $15 \mathrm{~min}$ with a No. 170 bur, and wet finishing was performed with silicone points (Politip F finishers and Politip P polishers, Vivadent, Schaan, Liechtenstein).

\section{Inadequate light}

The teeth were treated as in the control group, except that the light-curing unit was replaced with the inadequate light (68 $\mathrm{mW} / \mathrm{cm}^{2}$ ).

\section{Incomplete evaporation of primer solvent}

The teeth in this group were treated as in the control group, except that the primers were not dried prior to light curing and subsequent application of the dentin-enamel bonding resin. Light curing was performed by the same curing unit as in the control group $\left(420 \mathrm{~mW} / \mathrm{cm}^{2}\right)$.

\section{Microleakage}

The teeth were thermocycled between water baths of $5^{\circ} \mathrm{C}$ and $55^{\circ} \mathrm{C}$, with $30 \mathrm{sec}$ of dwell time in each bath. A total of 500 cycles was used for all specimens. After the thermal cycling, the specimens were stored in distilled water at $37^{\circ} \mathrm{C}$ for 7 days prior to being tested for microleakage. Cavities were cut in the root apices of each tooth, which were then filled with Vitremer (3M Co., St. Paul, MN). The entire tooth, except for the restoration and $1 \mathrm{~mm}$ of the tooth surface adjacent to the restoration, was coated with nail varnish. The teeth were then placed in a 50 wt $\%$ silver nitrate aqueous solution for $2 \mathrm{hr}$ in total darkness (Wu et al., 1983). Following retrieval, they were rinsed in running distilled water for $30 \mathrm{~min}$, immersed in photodeveloping solution, and exposed to a fluorescent light for $6 \mathrm{hr}$ so that silver ion reduction would be completed. After removal from the developing solution, the teeth were rinsed thoroughly in running water and immersed in acetone for 20 min to dissolve the layer of nail varnish.

\section{Clearing protocol}

Ten teeth from each group were randomly selected for the clearing protocol. The specimens were demineralized by immersion in Kristensen's fluid (1N sodium formate in $8 \mathrm{~N}$ formic acid, $\mathrm{pH}=2.2$ ). Radiographs of the specimen were taken to determine the end point of complete demineralization. The demineralized samples, now devoid of enamel, were washed 
thoroughly in running water for $2 \mathrm{hr}$. The specimens were dehydrated by passage through an ascending ethanol series $(70 \%, 80 \%, 95 \%)$, ending in 3 sequential changes in absolute ethanol. The opaque, completely demineralized and dehydrated specimens were then cleared by immersion in methyl salicylate (Merck, Darmstadt, Germany). Clearing was continued by further changes in fresh methyl salicylate until a faint amber, completely transparent, specimen was obtained, thus permitting an unobstructed view of the remaining opaque restoration together with the stereoscopic microleakage pattern along the restorative interface. Unrelated tooth structures and pulpal tissues were hand-trimmed with a razor blade, and the extent of microleakage along the cervical margin was graded on a scale of 0 to 4 under an optical stereomicroscope (Nikon, Stereo SMZ-10, Japan), based on a modified grading system for stereoscopic evaluation (Tay et al., 1994b) (Table 1).

\section{Scanning electron microscopy}

The remaining five undecalcified specimens from each group were used to determine the pathway of microleakage along the tooth-restorative interface. Three plano-parallel buccallingual sections were obtained from each specimen by a modified diamond saw under copious water supply. The sections were examined under a stereomicroscope, and the surface with the interface that exhibited the greatest extent of silver staining was selected for SEM examination. They were polished wet by 1200 -grit silicon carbide paper and etched with $10 \%$ phosphoric acid for $3 \mathrm{sec}$ to remove the smear layer and bring the interface into relief. The sections were then airdried, mounted onto brass stubs, conductively coated with carbon, and examined in a scanning electron microscope (JXA-840, JEOL, Japan) operating at 10-15 kV. Images were recorded in pairs by the secondary electron (SEI) and backscattered electron (BSE) modes.

\section{Results}

\section{Extent of microleakage}

The microleakage scores observed from the three groups are given in Table 2. A nonparametric one-way analysis of variance (Kruskal-Wallis test) showed a significant difference
Table 1. Three-dimensional microleakage scoring system along the cervical margin

\begin{tabular}{cc}
\hline Cervical Margin & Three-dimensional Leakage Score \\
\hline 0 & $\begin{array}{c}\text { No leakage from anywhere along the } \\
\text { circumference of the restoration } \\
\text { Anywhere along the circumference of } \\
\text { the restoration with silver } \\
\text { penetration extending up to one- } \\
\text { third of the cavity depth } \\
\text { Anywhere along the circumference of } \\
\text { the restoration with silver } \\
\text { penetration up to two-thirds of the } \\
\text { cavity depth } \\
\text { Anywhere along the circumference of } \\
\text { the restoration with silver } \\
\text { penetration greater than two-thirds } \\
\text { of the cavity depth, but not extending } \\
\text { into the axial wall } \\
\text { Anywhere along the circumference of } \\
\text { the restoration with silver } \\
\text { penetrating the axial wall }\end{array}$ \\
&
\end{tabular}

in the extent of leakage among the three groups $(\mathrm{H}=24.16$; $\mathrm{p}$ $<0.001)$. A post hoc nonparametric multiple-comparison procedure (Dunn test) (Glantz, 1992) further indicated that the groups differed significantly by pairwise comparison $(\mathrm{p}<$ 0.05) (Table 3).

Fig. 1 shows the typical extent of leakage (when present) that occurs in the control group when the primers were completely dried and light-cured with an adequate light source. The leakage observed is minimal and localized to discrete "islands" along the cavosurface margin. Fig. 2 shows a typical leakage pattern observed in the inadequatelight group when the primer was completely dried, but light-cured with an inferior light source. This appearance contrasts strikingly with that occurring in the "incomplete evaporation of primer solvent" group (Fig. 3), even though the primer mixture was light-cured with an adequate light

Table 2. Microleakage scores for individual samples recorded at the cervical margin

\begin{tabular}{|c|c|c|c|}
\hline Specimen & $\begin{array}{l}\text { Control } \\
\text { Primer completely dried } \\
\text { Adequate light source }\end{array}$ & $\begin{array}{l}\text { Inadequate Light } \\
\text { Primer completely dried } \\
\text { Inadequate light source }\end{array}$ & $\begin{array}{c}\text { Incomplete Evaporation } \\
\text { of Primer Solvent } \\
\text { Primer incompletely dried } \\
\text { Adequate light source }\end{array}$ \\
\hline 1 & 0 & 2 & 4 \\
\hline 2 & 1 & 2 & 4 \\
\hline 3 & 0 & 2 & 3 \\
\hline 4 & 0 & 1 & 4 \\
\hline 5 & 0 & 3 & 4 \\
\hline 6 & 1 & 2 & 4 \\
\hline 7 & 0 & 1 & 3 \\
\hline 8 & 0 & 2 & 4 \\
\hline 9 & 1 & 1 & 4 \\
\hline 10 & 1 & 2 & 2 \\
\hline
\end{tabular}


Table 3. Summary of nonparametric multiple comparisons against a single control-Dunn test

\begin{tabular}{lllll}
\hline Comparison & $\begin{array}{l}\text { Difference } \\
\text { of Ranks }\end{array}$ & $\begin{array}{l}\text { Standard } \\
\text { Error }\end{array}$ & $\mathrm{Q}$ & $\begin{array}{l}\text { Significance } \\
(\mathrm{P}<0.05)\end{array}$ \\
\hline
\end{tabular}

Incomplete

evaporation

of primer

solvent

$$
25.00-6.10=18.90
$$

Significant

vs.

Control

Incomplete

evaporation

of primer

solvent

$25.00-15.40=9.60$

3.85

2.497

vs.

Inadequate light

Inadequate light

vs.

Control

$15.40-6.10=9.30$

2.419 Significant source. Severe leakage can be observed along the entire cervical margin, extending into the axial wall of the resin composite restoration.

\section{Pathway of microleakage}

Microleakage at the etched enamel margin was virtually

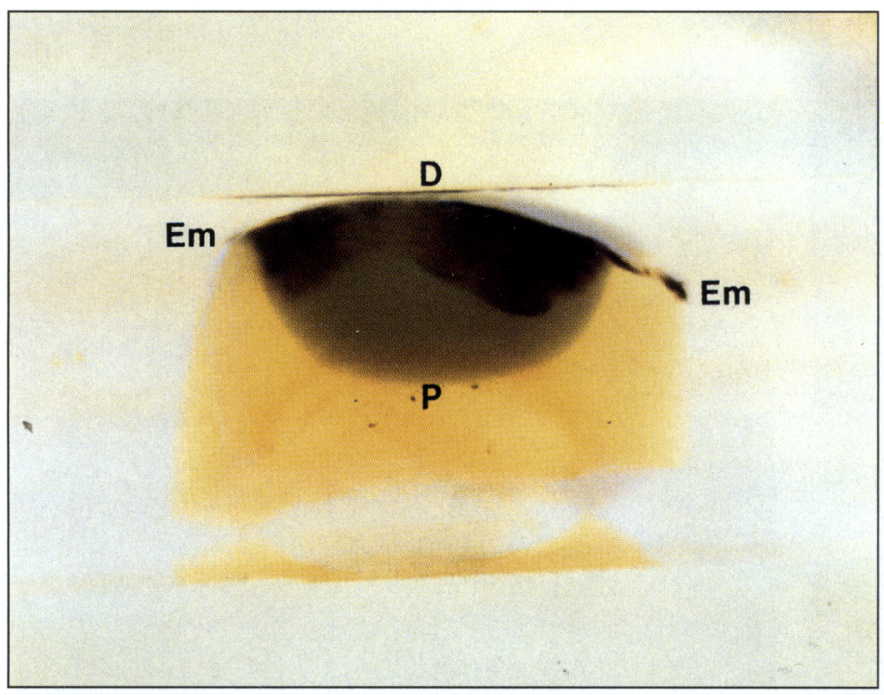

Figure 2. A typical example of the extent of microleakage in the inadequate-light group. Photograph of a specimen that has been cleared in methyl salicylate and observed from the cervical aspect. Silver has penetrated halfway along the cervical floor of the restoration. The depth of silver staining is not uniform; with deeper penetration toward the embrasure regions $(\mathrm{D}$, cervical cavosurface margin; $\mathrm{P}$, position indicating the location of the pulp, which has been removed after the clearing protocol for better illustration; Em, embrasure sides of the restoration).

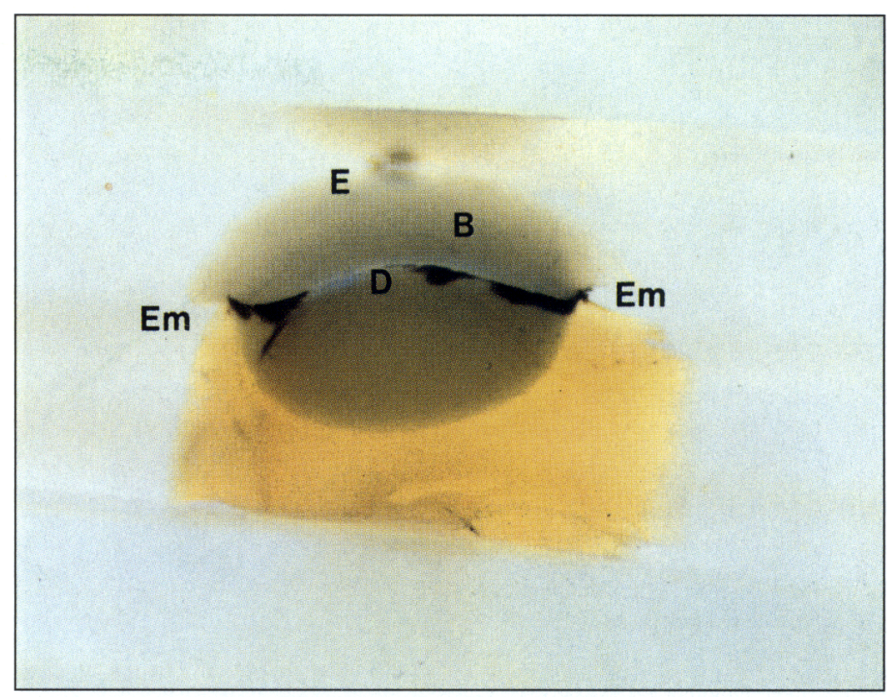

Figure 1. Extent of microleakage observed in the "control" group (adequate light, complete evaporation of primer solvent). Photograph of a specimen that has been cleared in methyl salicylate and observed from a cervical-oblique angle. Minimal, isolated areas of silver staining are present along the cervical cavosurface margin (E, occlusal cavosurface margin; D, cervical cavosurface margin; B, buccal surface of the cylindrical restoration; Em, embrasure sides of the restoration).

absent from the control group (Figs. 4a,b). However, each group did permit some microleakage when margins were placed in either dentin or cementum. Five different patterns, with increasingly severe microleakage, could be recognized:

Zone $A-$ No leakage is present anywhere along the toothrestorative interface (Figs. 5a,b). Included in this category

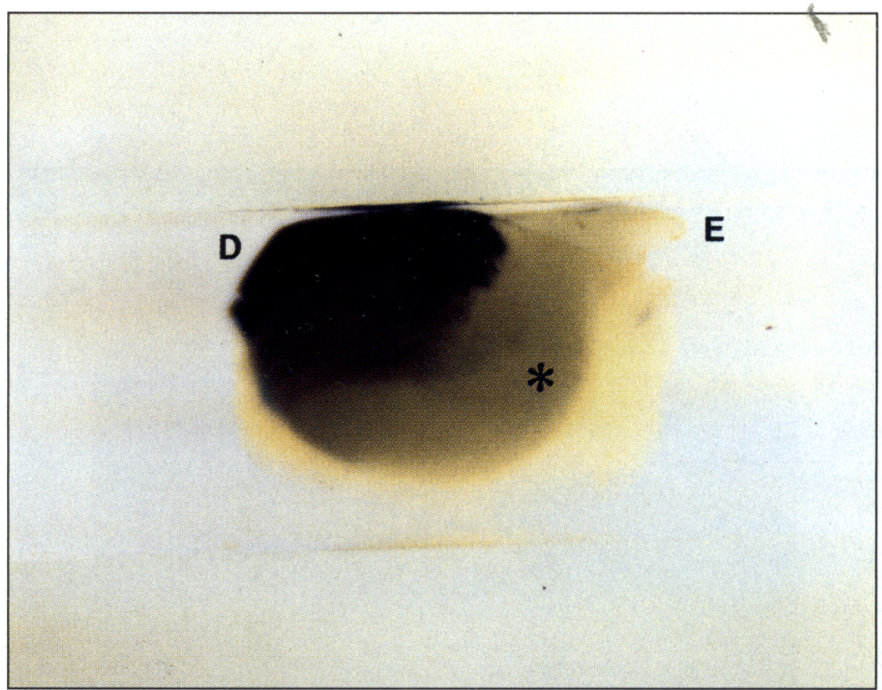

Figure 3. Extent of microleakage observed in the "incomplete evaporation of primer solvent" group. Photograph of a specimen that has been cleared in methyl salicylate and observed from the embrasure aspect of the restoration. No silver staining is observed along the occlusal side of the restoration, which was previously covered with enamel. However, extensive silver staining is found along the cervical margins, extending all the way into the axial wall of the cavity preparation (E, occlusal cavosurface margin-a bevel can be observed in the part of the restoration which was previously covered with enamel; D, cervical side of the restoration; asterisk, indicates the pulpo-gingival line angle of the cavity preparation). 

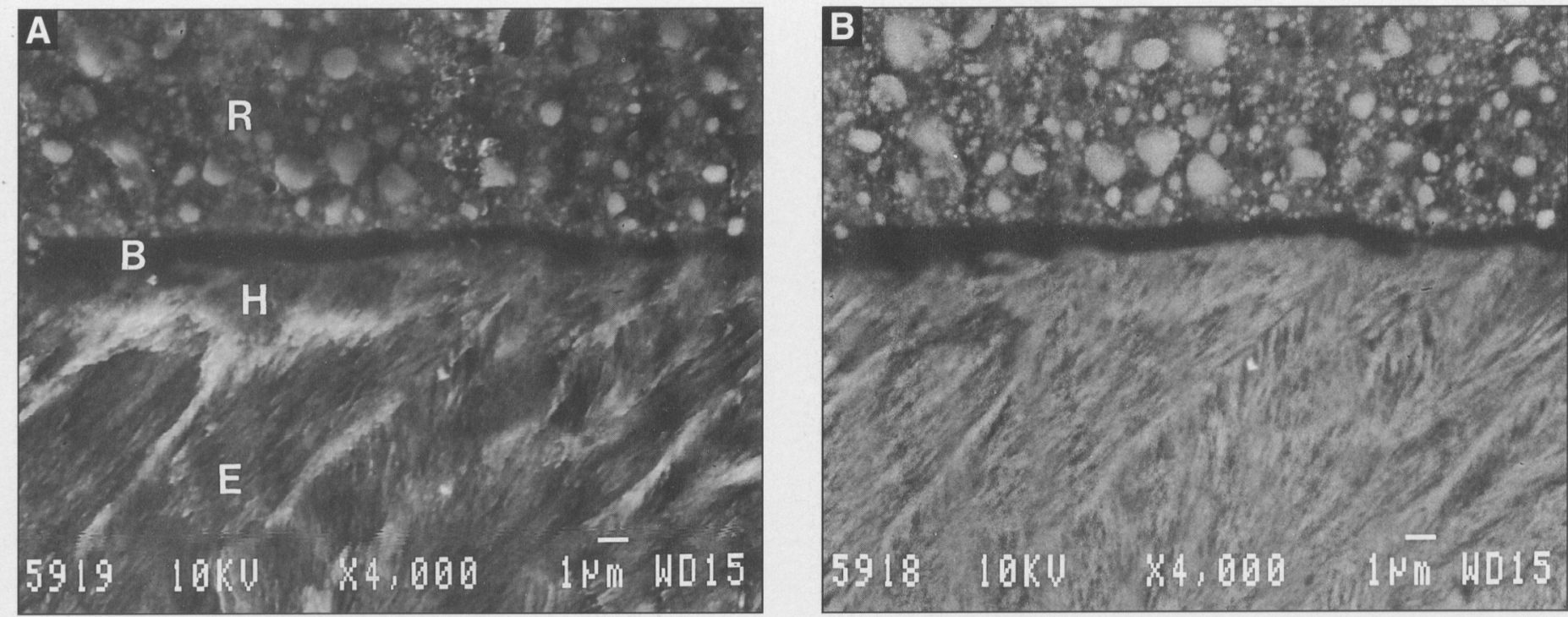

Figure 4. Scanning electron micrograph of the tooth-restorative interface when the margin is placed in enamel. No silver staining or gap is present. (A) Secondary electron mode. (B) Back-scattered mode. R, resin composite, Z100 with spherical fillers; B, bonding resin; H, resinimpregnated enamel; E, enamel prism.

are regions that exhibit artifactual gaps with the absence of bright-staining silver granules along the gap margins.

Zone $B$-Leakage is localized along the interface between the hybrid layer and the bonding resin. A discrete line, consisting of bright spots of an element of high atomic weight (in this case, silver), can be observed in the BSE mode (Figs. 6a,b).

Zone C-Leakage is present within the hybrid layer as well as the bonding resin (Figs. 7a,b). The interface between the hybrid layer and the bonding resin, however, is still intact.
Zone $D$-More extensive leakage can be seen within the hybrid layer and the bonding resin. Separation of the hybrid layer and bonding resin interface is observed, as demonstrated by the presence of silver grains along the gap margin (Figs. 8a,b).

Zone E-Incomplete evaporation of the primer solvent produces a beaded appearance along the interface (Fig. 9a). Gap formation is evident, together with extensive leakage within the bonding resin and hybrid layer and extending into the dentinal tubules (Fig. 9b).

The distribution of the various zones within the three
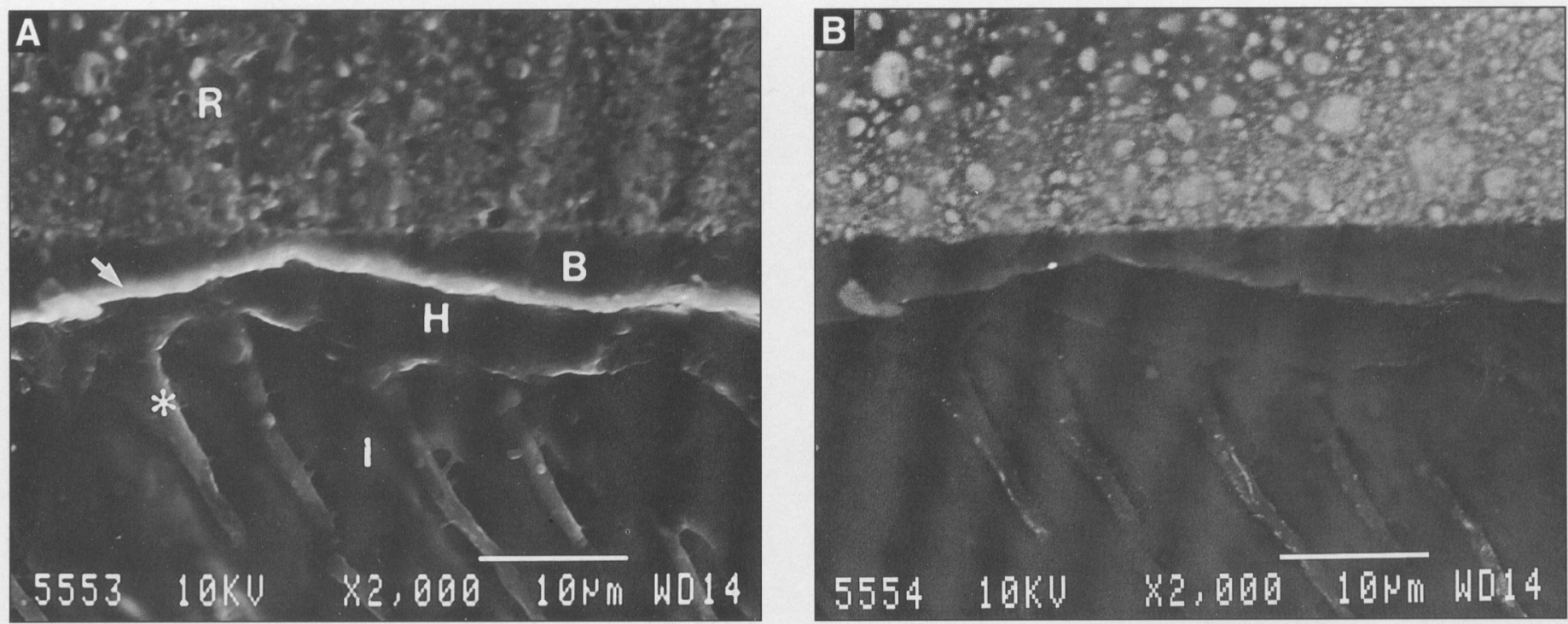

Figure 5. Pathway of microleakage along the dentin-restorative interface is characterized by five different patterns (Zones A to E). Scanning electron micrographs of Zone A of the dentin-restorative interface. No silver staining or gap is present. (A) Secondary electron mode. (B) Back-scattered mode. The intense white line (arrow) along the hybrid layer-bonding resin interface in the SEI mode is a topographical edging effect and is absent from the BSE mode. R, resin composite; B, bonding resin; H, resin-impregnated dentin; I, intertubular dentin; asterisk, resin tag. 

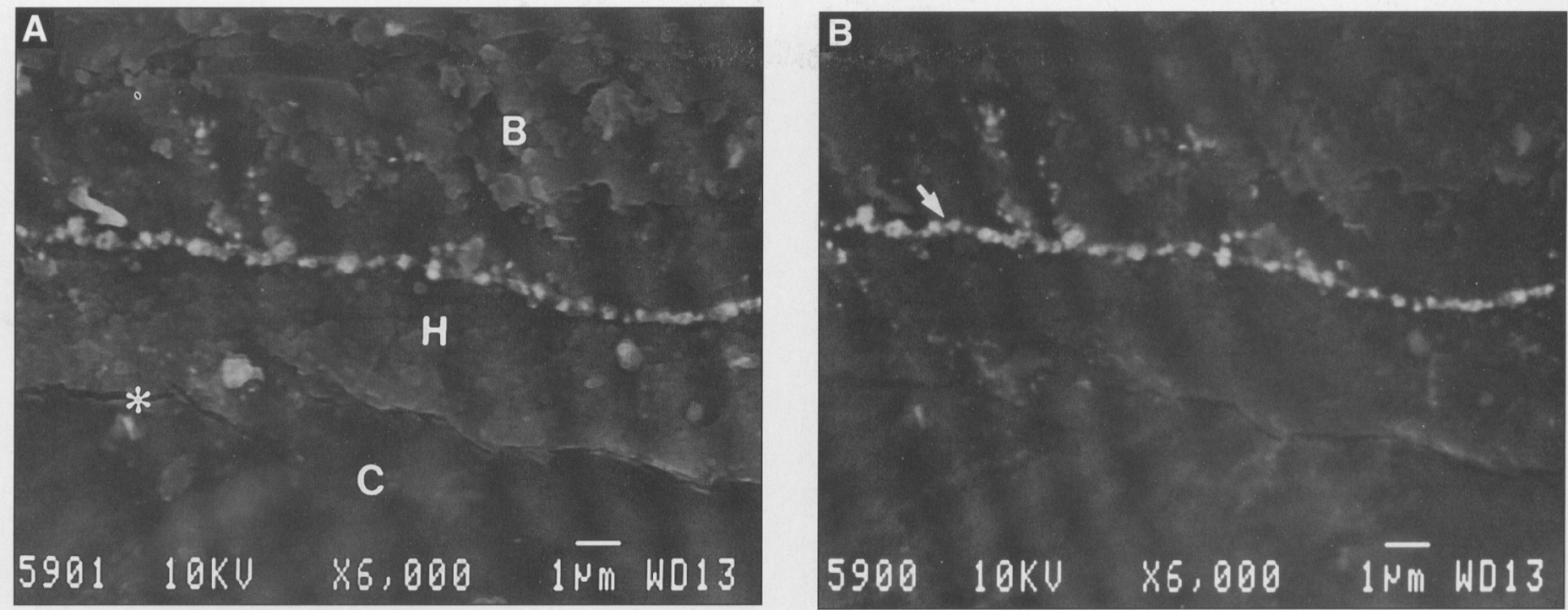

Figure 6. Scanning electron micrographs of Zone B of the cementum-restorative interface. Silver staining is restricted to the interface between the bonding resin and hybrid layer (arrow). Note the absence of interfacial gap. (A) Secondary electron mode. (B) Back-scattered mode. B, bonding resin; $\mathrm{H}$, resin-impregnated cementum; C, cementum; asterisk, artifactual gap without silver staining.

groups is presented in Fig. 10. For ease of description, they were reported independently under a particular group, commencing with no leakage (Zone A) to the most extensive leakage pattern (Zone E). In reality, a section within a particular group that harbored the more severe pattern always encompassed the milder patterns further from the site depicted. In addition, silver staining was also detected along the sites of incremental resin composite placement in the inadequate-light group.

\section{Discussion}

The interactive use of a clearing protocol and SEM examination of the interface provides a clear portrait of the extent and pathway of microleakage. The clearing protocol has been shown to be an effective and non-labor-intensive technique for stereoscopic evaluation of the extent of microleakage along the dentin-restorative margin (Tay et al., 1994b). The silver staining method permits the microleakage pathway to be studied by secondary electron and backscattered scanning electron microscopy (Wieczkowski et al., 1992). Secondary electron images provide essentially topographical information of the interface, while backscattered electron images also reveal complementary information on elemental variation, the intensity of which is partly determined by the increasing atomic number of the scattering atoms within the specimens (Watt, 1985). Cryo-
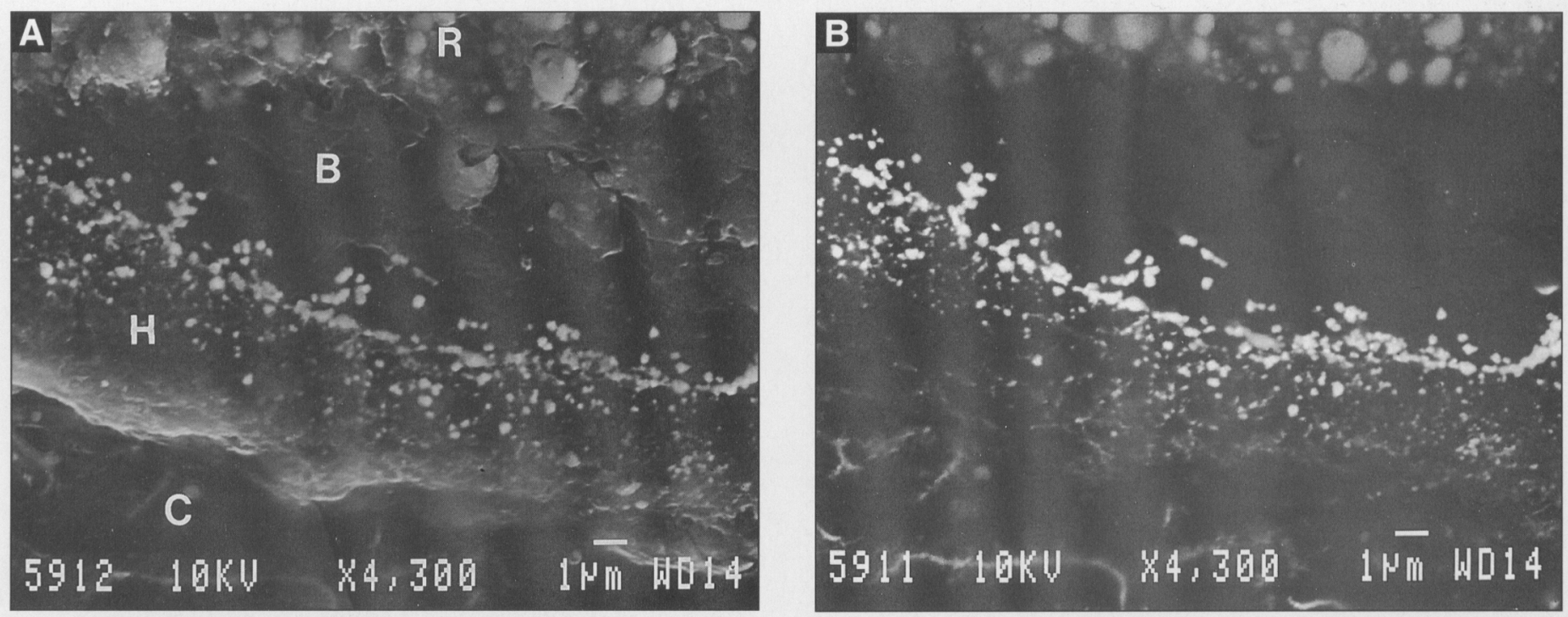

Figure 7. Scanning electron micrographs of Zone $C$ of the cementum-restorative interface. Silver staining is present along the bonding resinhybrid layer interface as well as within the two regions. No visibly discernible interfacial gap is observed. (A) Secondary electron mode. (B) Back-scattered mode. R, resin composite; B, bonding resin; H, resin-impregnated cementum; C, cementum. 

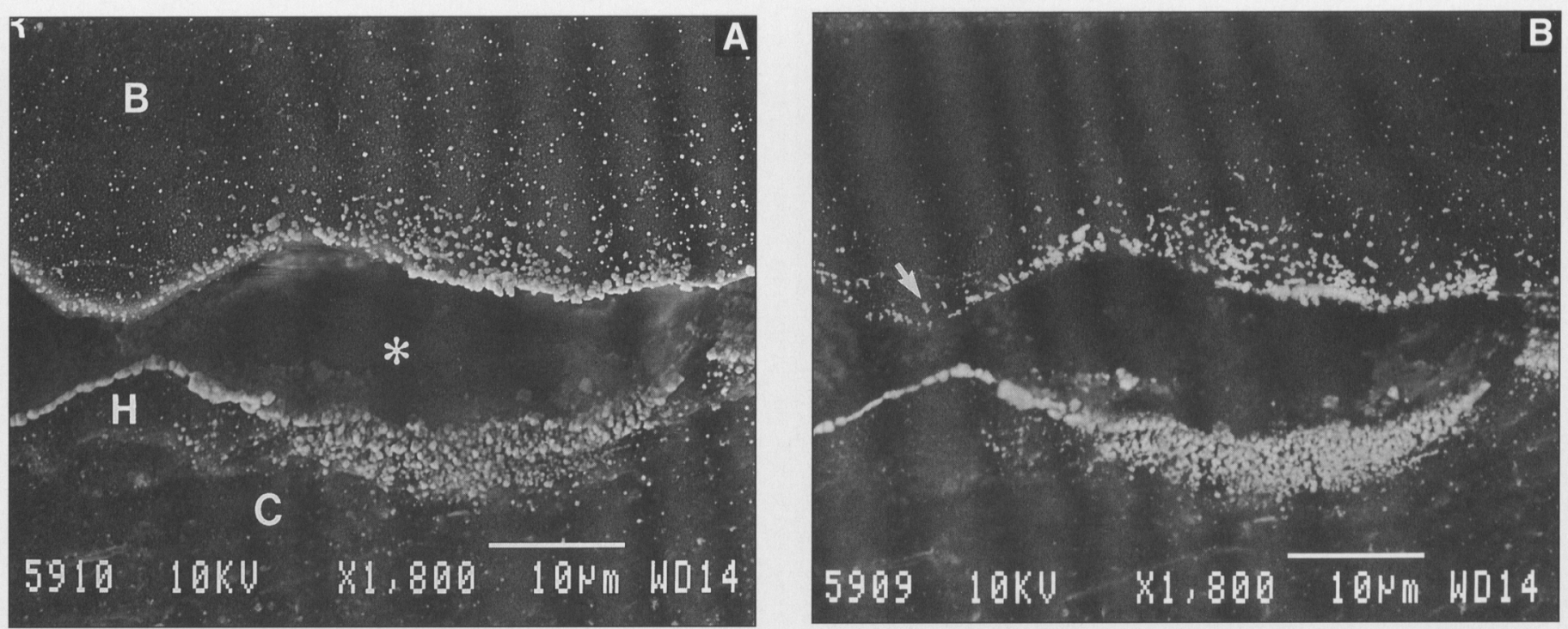

Figure 8. Scanning electron micrographs of Zone D of the cementum-restorative interface. Silver staining is more severe within the hybrid layer and bonding resin. Silver grains were also found to line the edges of the interfacial gap (arrow). (A) Secondary electron mode. (B) Backscattered mode. R, resin composite; B, bonding resin; asterisk, interfacial gap; $\mathrm{H}$, resin-impregnated cementum; $\mathrm{C}$, cementum.

SEM has been used to distinguish true from artifactual gaps created upon dehydration during specimen preparation for conventional SEM observation (Sano et al., 1994a). Such artifacts, however, can be easily differentiated from true gaps by the absence of silver staining along the gap border, when specimens are observed in the back-scattered electron mode (Figs. 6a,b). The recognition of the higher atomic number tracer element is often concealed by an edge effect when topographical features are revealed under the secondary electron mode as a result of the higher secondary electron yield around edges and corners (Figs. 8a,b).

The absence of leakage in the control group when the restorative margin is bounded by enamel (Fig. 4b) confirms the stability and tenacity of the enamel bond produced by acid conditioning and clearly indicates the clinical superiority of such a situation whenever it can be attained. In addition, the presence of a layer of resin-infiltrated enamel (Fig. 4a) further confirms the hybridization concept that was originally observed and described in enamel (Gwinnett and Matsui, 1967).

None of the three groups evaluated in this in vitro study completely eliminated microleakage along the dentin/cementum margins. All-Bond 2 had been previously reported to exhibit significantly less microleakage than Scotchbond Multi-Purpose, with a median leakage score of " 1 " for both bulk and incremental filling methods when the
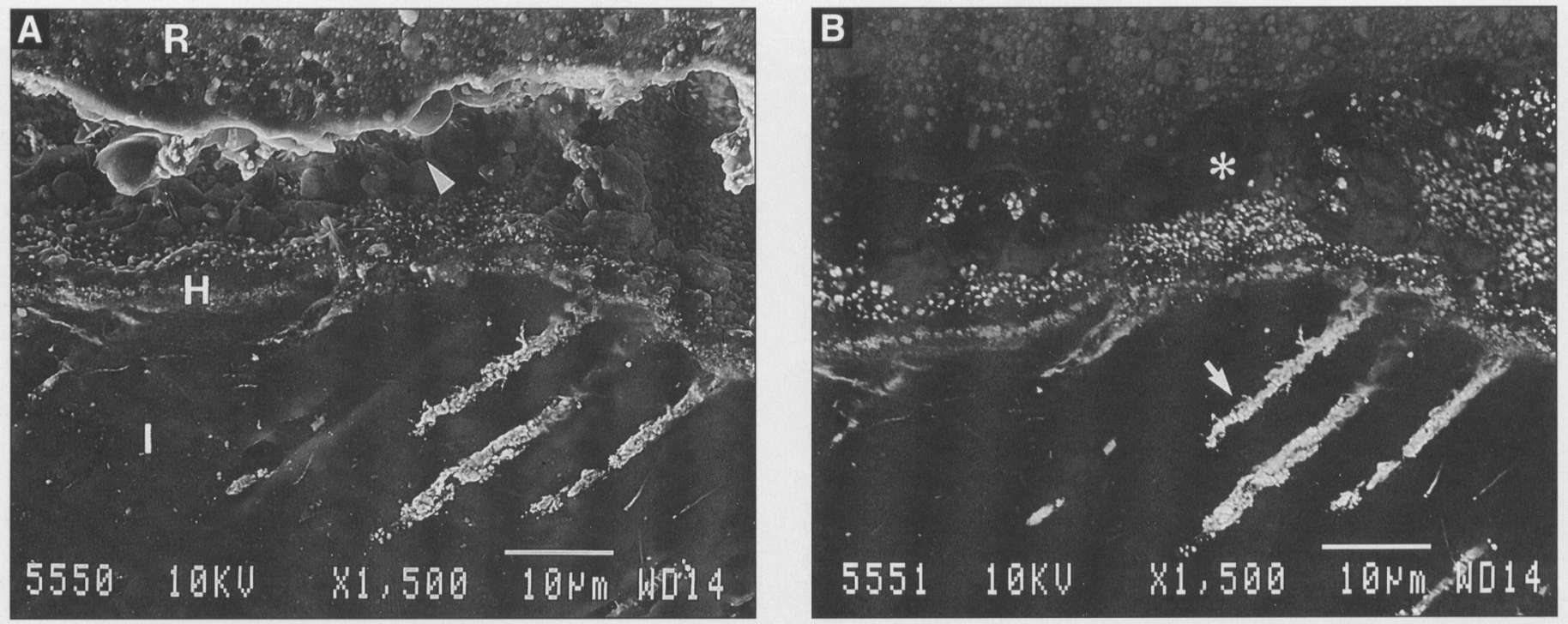

Figure 9. Scanning electron micrographs of Zone E of the dentin-restorative interface. The bonding resin layer and the hybrid layer are incomplete and assumed a beaded appearance (arrowhead). Silver penetration into the dentinal tubules is evident (arrow). (A) Secondary electron mode. (B) Back-scattered mode. R, resin composite; H, resin-impregnated dentin; I, intertubular dentin; asterisk, interfacial gap. 


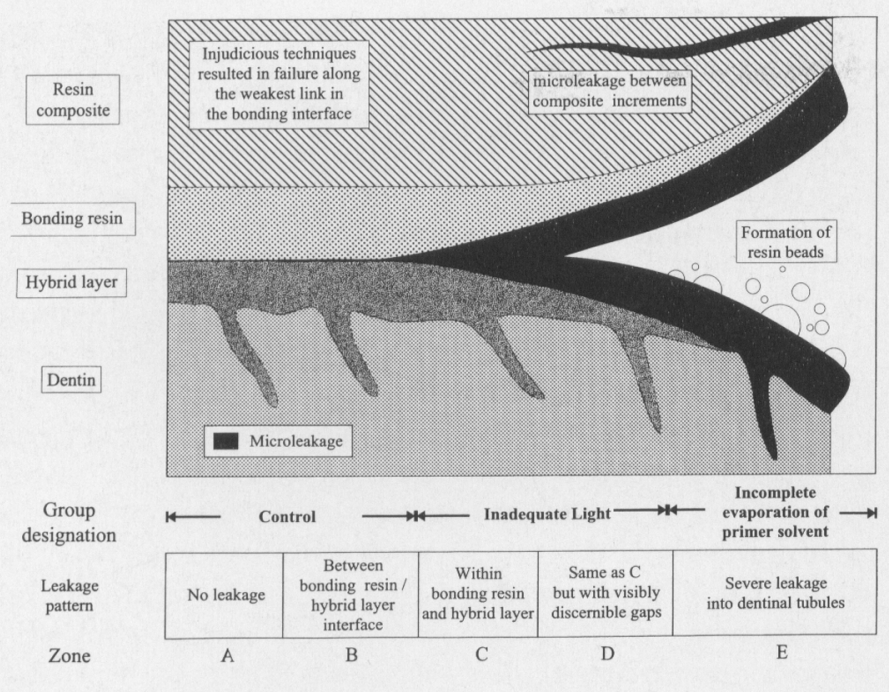

Figure 10. A schematic represention of the relative distribution of the five microleakage zones within the three groups. The observation of a more severe pattern is always preceded by other milder leakage patterns. The interface between the bonding resin and hybrid layer depicts the weak link in the bonding assembly.

All-Bond 2 primers A and B were chemically cured only (Linden and Swift, 1994). The results of the present study suggest that the extent of leakage for the All-Bond 2 system might be even less when the primers are applied under optimal conditions (completely dried and light-cured). Furthermore, the extent of leakage is not uniform along the entire dentin/cementum margin and appears to be concentrated around the cemento-enamel junction (i.e., the embrasure regions).

The newer generation of adhesives emphasizes its potential to create a hybrid layer (resin-impregnated dentin) between resin and dentin (Inokoshi et al., 1993). Such systems invariably involve the use of a dentin/enamel conditioner, a primer, and a bonding resin, followed by the placement of a resin composite, resulting in an increase in the number of interfaces between the restoration and the tooth substrate. We hypothesize that, in a system that uses a total-etch technique to remove the smear layer completely and partially demineralize the underlying dentin, microleakage may occur in any, or a combination, of at least eight regions within the dentin-restorative junction (Fig. 11, nos. one to eight). No attempt is made to differentiate the primer resin from the dentin-enamel bonding resin, since they were not discernible from one another in the present study, which utilized an acidrinse technique to bring the various interfaces into relief, and the two are collectively termed "bonding resin" in the schematic representation. Differentiation between the primer and bonding resin has not been reported in another scanning electron microscope study that usèd argon-ion milling to remove the smear layer and enhance the surface relief following specimen polishing (Van Meerbeek et al., 1992), as well as in transmission electron microscope studies (Eick et al., 1993b; Van Meerbeek et al., 1993).

Examination of the microleakage pathway in the three groups reveals that while an array of progressive severities might occur in a particular group, the site of microleakage

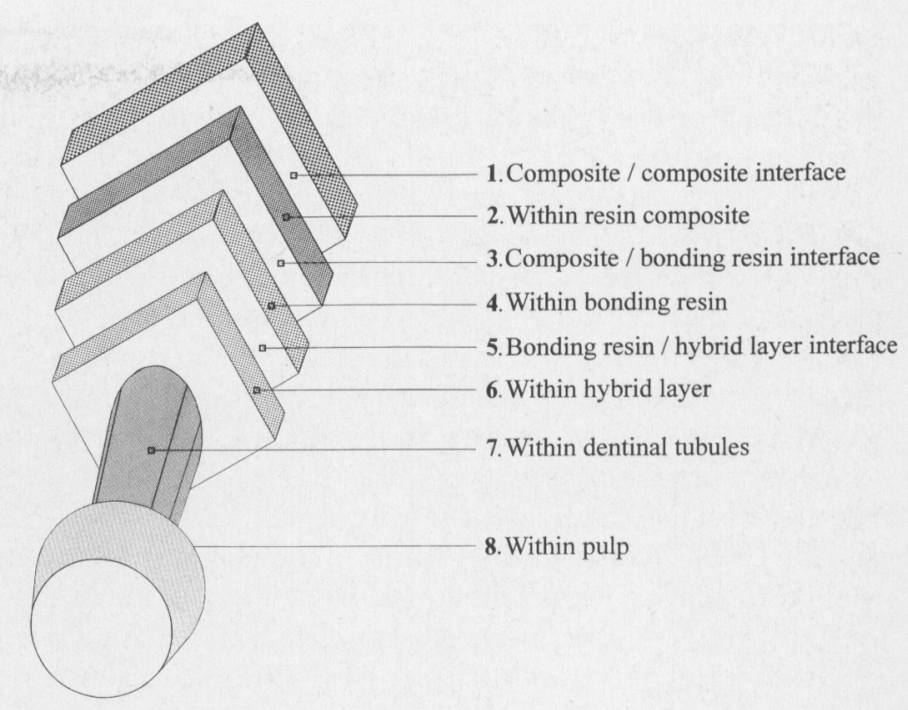

Figure 11. Possible sites (or combinations) whereby microleakage may occur along the tooth-restorative interface in a total-etch dentin adhesive. These sites are numbered 1 to 8 in the schematic. The primer and bonding resin are grouped into a single category, since they are not discernible from one another under the present protocol.

initiation is identical in all three groups and appears to originate from the interface between the bonding resin and hybrid layer (Fig. 6b). The bond between a restoration and tooth substrate might be equated with a chain, comprised of a series of links, which could be only as strong as its weakest link (Gwinnett, 1988). From the present study, the weak link in the All-Bond 2 system is the resin-hybrid layer interface. A scanning microscope (SEM) fractographic study of Clearfil Linerbond, Super Bond D-liner, and Resto Bond 3 revealed the persistence of the resin-impregnated (hybrid) layer on the dentin surface, in spite of the difference in the bonding system and age of dentin, indicating that adhesive failure occurred initially at the interface between the bonding resin and the hybrid layer (Tagami et al., 1993). A study on the effect of long-term storage on specimens bonded with Amalgambond and All-Bond 2 revealed similar results (Gwinnett and $Y u, 1994)$. Failure analysis of enamel-dentin crown fragments re-attached, following crown fracture, with All-Bond 2 and Scotchbond Multi-Purpose showed that the weak link in the bond between tooth fragment and remaining tooth structure was the bonding resin (Andreasen et al., 1993). Such information, supported by the microleakage data derived from the present study, was not in agreement with the hypothesis that degradation of the incompletely resin-infiltrated, demineralized collagen fibers within the base of the hybrid layer constituted the weak link in current total-etch dentin adhesive systems (Nakabayashi et al., 1991; Pashley et al., 1993). While the above statement may be true for other dentin adhesive systems, energy dispersive x-ray micro-analysis (EDS) with a sulphur analog for hydroxyethylmethacrylate (HEMA) in Scotchbond Multi-Purpose (Eick et al., 1993a) and a bromine analog for biphenyldimethacrylate (BPDM) in the All-Bond 2 wetbonding technique (Gwinnett and Tay, unpublished observations) indicated that the primers in these current total-etch systems wetted and permeated the demineralized 
dentin very effectively.

Curing of the primer with an inadequate light after evaporation of the primer solvent results in the localization of silver staining along the interface as well as within the hybrid layer and bonding resin. Such a distribution is initially observed in areas devoid of visibly discernible gaps (Fig. $7 b$ ) and in more distant areas, where such interfacial gaps can be identified (Fig. 8b). The current version of the All-Bond 2 primers is a dual-cure system. The quantity of unreacted remaining double bonds (RDB) in restorative resin polymers in a light-curing material was found to be relatively small compared with that present in chemically cured materials of adequate setting time (Asmussen, 1982; Ruyter, 1985). Likewise, the chemical component of cure in dual-cure resin cements was found to be always lower than the light-cured component (Rueggeberg and Caughman, 1993). Fourier transform infrared (FTIR) spectroscopic analysis showed that the degree of monomer conversion in the unfilled All-Bond 2 primers was higher when they were light-cured than when chemically cured alone (Suh, personal communication). Monomer conversion (Rueggeberg et al., 1993) and polymerization contraction (Sakaguchi et al., 1992) of light-activated resin composites were found to be related to light intensity and length of exposure.

Microleakage within the hybrid layer has previously been observed in the absence of interfacial gaps in various dentin adhesives and was attributed to incomplete penetration of resin monomers into decalcified dentin created by phosphoric acid etching (Sano et al., 1994a,b). The findings in the present study, however, did not agree with this explanation. Examination of the sections from the inadequate-light group (Figs. 7,8 ) revealed that the presence of silver staining within the hybrid layer or bonding resin is always accompanied by a discrete line of silver grains along the interface, regardless of the presence or absence of interfacial gaps. Furthermore, those familiar with silver nitrate microleakage studies would invariably notice an initial zone of around 50 to $150 \mu \mathrm{m}$ where silver nitrate has penetrated the dentinal tubules or cementum through the "window" created between the nail varnish and the restoration. This artifactual zone generally terminates without silver penetrating all the way through and blackening the entire tooth segment covered by those tubules (Linden and Swift, 1994). It is debatable how silver ions could have traversed an external surface, through the porosities within an incompletely infiltrated hybrid layer, in the absence of interfacial gaps, and be deposited far from the external surface of the restoration. It is postulated that the weak link along the resin-hybrid layer interface acts as an initial site whereby movement of extraneous substances such as water molecules occurs. This may result in water sorption of the incompletely converted monomers, resulting in long-term deterioration of the partially polymerized resin materials within the hybrid layer and bonding resin. This hypothesis is supported by another study showing that, on long-term storage of Super Bond D-liner bonded specimens over a one-year period, the adhesive failure that was always observed initially at the tooth interface was subsequently accompanied by failure within the hybrid layer itself (Burrow et al., 1993).
The observation of a zone of resin-impregnated cementum (Figs. 6, 7, 8) in the present study requires further micromorphological investigation. In the present study, such a zone is devoid of resin tags, in contrast to the zone of resinimpregnated dentin, which is traversed by resin tags even if tubules are running almost parallel to the surface of the cavity preparation. Little is reported in the literature of the interaction of dentin adhesives with partially or totally demineralized cementum. The majority of microleakage studies on dentin adhesives and glass-ionomer cements regards the dentino-cemental junction as behaving like dentin in relation to the restorative sealing properties and possible marginal leakage. A scanning electron microscope study of extracted teeth with Class $\mathrm{V}$ cavity preparation showed that well-developed cementum margins could be produced, and that the susceptibility of marginal leakage might be related to the restorative material used and whether acid-etch techniques were involved (Hargreaves et al., 1989). Katoh and Shinkai (1989) reported a difference in the degree of gap formation along the gingival cementum wall and the gingival dentin wall, when a total-etch technique was used on cervical cavities restored with Clearfil Bond System F. The ultrastructure of exposed cementum below the cemento-enamel junction has been found to differ from that of inner sound cementum (Kockapan, 1990). Since the dental treatment needs of many geriatric patients include root surface caries and Class V restorations involving exposed cementum, further studies of this most peripheral marginal seal would be useful.

Topographical features of an incompletely resininfiltrated hybrid layer and/or bonding resin together with the presence of extensive leakage into the dentinal tubules (Fig 9) indicate that incomplete evaporation of the primer solvent, even in the presence of adequate light curing, is detrimental to the attainment of a hermetic seal. The primer A in the All-Bond 2 system contains $17.4 \%$ ethanol and $17.4 \%$ water in the solvent (Suh, personal communication). The presence of unevaporated ethanol or water may interfere with the polymerization of the primers and/or bonding resin, resulting in a beaded appearance (Fig. 9a, arrowhead). These resin beads appear to be similar to the intratubular resin globules previously observed in vivo for the same adhesive system (Tay et al., 1994a), although they are much larger and are located on the dentin surface. The registration of significant differences when the three groups are compared pairwise suggests that complete evaporation of the primer solvent makes a more significant contribution than light curing in maintaining the marginal integrity of the dentin-restorative junction. Further investigations are being conducted in our laboratories regarding the exact nature of these extraneous resin beads.

The pathways of microleakage observed in the three groups represent a sequence of ascending severity that is expedited by the weak link in the bonding resin-hybrid layer interface. The presence of silver staining along this interface (Zone B) suggests that contractional stresses during polymerization of the resin composite have already created a separation of the margins. The absence of an interfacial gap, on the other hand, may be due to hygroscopic expansion of the resin composite which may eventually compensate for the polymerization contraction (Torstenson 
and Brännström, 1988; Davidson and Kemp-Scholte, 1989). Water sorption of unfilled methacrylate-based copolymers has been estimated to vary between 3 and $6 \%$, depending on the proportion of the diluent (Kalachandra and Turner, 1987). Water sorption by the suboptimally converted, unfilled primer and bonding resins may also account for the closure of the interfacial gap at Zone $\mathrm{C}$. This represents the borderline of a gap-free, clinically acceptable restoration that is free from sensitivity and with the dentinal tubules sealed intact. Any marginal staining, being so minute at this stage, would have evaded the scrutiny of the naked eye.

Hygroscopic expansion was rarely sufficient to compensate completely for polymerization shrinkage (Bowen et al., 1982). This is manifested by separation of the interface at Zone D, resulting in the appearance of interfacial gaps. While the tubules are still sealed intact, marginal staining within the gaps will be an eventual clinical consequence. Furthermore, flexure of the restored teeth during occlusal stresses may create further abfractions within the region (Grippo, 1992), widening the original gap, which has been compensated for by hygroscopic expansion. Failure of the primer solvent to evaporate thoroughly presents the worst scenario, with characteristic interfacial defects which are detectable under the SEM, together with severe leakage into the tubules, allowing the movement of fluid and bacteria through the interface and into the tubules. Such failures will be manifested clinically as post-operative sensitivity and recurrent caries.

Previous studies have demonstrated that the totaletching technique is more effective than etching of the occlusal enamel alone in reducing microleakage along the cervical wall of Class $\mathrm{V}$ resin composite restorations (Fusayama and Kohno, 1989; Katoh and Shinkai, 1989). The present study supports these and other reports that gap-free restorations can be produced with the use of a total-etch technique and acetone-containing dentin adhesives (Gwinnett and Kanca, 1992a,b; Gwinnett and Yu, 1994). This study further shows that the physical existence or nonexistence of gaps is not an absolute phenomenon but is represented by an array of progressively deleterious microleakage patterns. The potential to seal off exposed dentinal tubules represents a dramatic improvement over previous generations of dentin adhesives (Swift, 1991; Linden and Swift, 1994). However, the ability to render a truly non-leaking restoration beyond the cemento-enamel junction still depends on how the adhesive performs in resisting polymerization contraction, thermal, and occlusal loading stresses on the resin composite (Retief, 1994). This may be improved by a single primer-bonding resin version of the system. It would also be interesting to see if the surface resin beads can be eliminated through a reduction in either the water or alcohol content of the present system. On the other hand, the capability to produce a clinically gapfree restoration depends largely upon extrinsic factors forged by the skill of the clinician. While the necessity for good isolation (Christensen, 1992) and the avoidance of onebulk placement of resin composite (Tjan et al., 1992) cannot be overstated, the present study clearly shows that suboptimal monomer conversion, as exemplified by an inadequate light-curing unit, and, even more important, the failure of the primer solvent to evaporate completely can result in the presence of gaps along the tooth-restorative interface. Injudicious techniques as such may account for the persistence of post-operative sensitivity or the extensive microleakage reported in some studies. Preliminary clinical observations in which the conditioned dentin is free from visible surface moisture, with the primer thoroughly dried and adequately cured prior to the placement of the bonding agent and composite, support the conclusions drawn from this study. Post-operative sensitivity was rarely encountered.

\section{Acknowledgments}

This investigation was supported by the Committee on Research and Conference Grants (CRCG), the University of Hong Kong. The technical expertise of Lee Man Tung and Simon Lee of the Oral Biology Unit, and Sam Hui and Vicky Yip of the Dental Illustration Unit, Prince Philip Dental Hospital, the University of Hong Kong, is graciously acknowledged.

\section{References}

Andreasen FM, Steinhardt U, Bille M, Munksgaard EC (1993). Bonding of enamel-dentin crown fragments after crown fracture. An experimental study using bonding agents. Endod Dent Traumatol 9:111-114.

Asmussen E (1982). Factors affecting the quantity of remaining double bonds in restorative resin polymers. Scand J Dent Res 90:490-496.

Bertolotti RL (1990). Acid etching of dentin. Quintessence Int 21:77-88.

Bowen RL, Rapson JE, Dickson G (1982). Hardening shrinkage and hygroscopic expansion of composite resins. J Dent Res 61:654-658.

Brännström M, Linden LA, Anström A (1967). The hydrodynamics of the dentinal tubule and of pulpal fluid. A discussion of its significance in relation to dentinal sensitivity. Caries Res 1:310-317.

Burrow MF, Tagami J, Hosoda H (1993). The long term durability of bond strengths to dentin. Bull Tokyo Med Dent Univ 40:173-191.

Christensen GJ (1992). Clinical factors affecting adhesion. Oper Dent (Suppl 5):24-31.

Cox CF (1992). Microleakage related to restorative procedures. Proc Finn Dent Soc 88(Suppl 1):83-93.

Davidson CL, Kemp-Scholte CM (1989). Shortcomings of composite resins in Class V restorations. J Esthet Dent 1:1-4.

Davila JM, Gwinnett AJ, Robles JC (1988). Marginal adaptation of composite resins and dentinal bonding agents. J Dent Child 55:25-28.

Eick JD, Robinson SJ, Byerley TJ, Chappelow CC (1993a). Adhesives and nonshrinking dental resins of the future. Quintessence Int 24:632-640.

Eick JD, Robinson SJ, Chappell RP, Cobb CM, Spencer P (1993b). The dentinal surface: Its influence on dentinal adhesion. Part III. Quintessence Int 24:571-582.

Feilzer AJ, deGee AJ, Davidson CL (1988). Curing contraction of composite and glass ionomer cements. J Prosthet Dent 59:298-300.

Fusayama A, Kohno A (1989). Marginal closure of composite 
restorations with its gingival wall in cementum/dentin. $J$ Prosthet Dent 61:293-296.

Fusayama T (1992a). Indications for self-cured and light-cured adhesive composite resins. J Prosthet Dent 67:46-51.

Fusayama T (1992b). Total etch technique and cavity isolation. J Esthet Dent 4:105-109.

Garcia-Godoy F, Finger WJ (1993). Reliability of microleakage evaluation using dentin bonding agents (abstract). J Dent Res 72:308.

Glantz SA (1992). Primer of biostatistics. 3rd ed. New York: McGraw-Hill, Inc., pp. 320-371.

Grippo JO (1992). Noncarious cervical lesions: the decision to ignore or restore. J Esthet Dent 4(Suppl):55-64.

Gwinnett AJ (1988). Bonding of restorative resins to enamel. Int Dent J 38:91-96.

Gwinnett AJ (1992). Moist versus dry dentin: its effect on shear bond strength. Am J Dent 5:127-129.

Gwinnett AJ, Kanca J III (1992a). Micromorphological relationship between resin and dentin in vivo and in vitro. Am J Dent 5:19-23.

Gwinnett AJ, Kanca J III (1992b). Interfacial morphology of resin composite and shiny erosion lesions. Am J Dent 5:315-317.

Gwinnett AJ, Matsui A (1967). A study of enamel adhesives. The physical relationship between enamel and adhesive. Arch Oral Biol 12:1615-1618.

Gwinnett AJ, Yu S (1994). Effect of long-term storage on dentin bonding. Am J Dent 8:109-111.

Gwinnett AJ, Dickerson WG, Yu S (1992). Dentin bond shear strength and microleakage for Syntac/Heliomolar: a comparison between the manufacturer's and total etch technique. J Esthet Dent 4:164-168.

Hargreaves JA, Grossman ES, Matejka JM (1989). Scanning electron microscopic study of prepared cavities involving enamel, dentin, and cementum. J Prosthet Dent 61:191-197.

Inokoshi S, Hosoda H, Harnirattisai C, Shimada Y (1993). Interfacial structure between dentin and seven dentin bonding systems revealed using argon ion beam etching. Oper Dent 18:8-16.

Kalachandra S, Turner DT (1987). Water sorption of polymethacrylate networks: bis-GMA/TEGDM copolymers. J Biomed Mater Res 21:329-338.

Katoh Y, Shinkai K (1989). Study on microleakage and gap observation of composite resin restoration. Shigaku 77:712726.

Kockapan C (1990). Elektronenmikroskopische untersuchungen über die struktur des freiliegeden zementes (Electron microscopic research on the structures of exposed cementum). Schweiz Monatsschr Zahnmed 199:709-714 (in German).

Linden JF, Swift EJ (1994). Microleakage of two new dentin adhesives. Am J Dent 7:31-34.

Nakabayashi N, Nakamura M, Yasuda N (1991). Hybrid layer as a dentin-bonding mechanism. J Esthet Dent 3:133-138.

Pashley DH, Ciucchi B, Sano H, Horner JA (1993). Permeability of dentin to adhesive agents. Quintessence Int 24:618-631.

Retief DH (1994). Do adhesives prevent microleakage? Int Dent J 44:19-26.
Rueggeberg FA, Caughman WF (1993). The influence of light exposure on polymerization of dual-cure resin cements. Oper Dent 18:48-55.

Rueggeberg FA, Caughman WF, Curtis JW Jr, Davis HC (1993). Factors affecting cure at depths within light-activated resin composites. Am J Dent 6:91-95.

Ruyter IE (1985). Monomer systems and polymerization. In: International symposium on posterior composite resin dental restorative materials. Vanherle G, Smith DC, eds. St. Paul, MN: Minnesota Mining and Mfg. Co., pp. 109-135.

Sakaguchi RL, Douglas WH, Peters MC (1992). Curing light performance and polymerization of composite restorative materials. J Dent 20:183-188.

Sano H, Shono T, Takatsu T, Hosoda H (1994a). Microporous dentin zone beneath resin-impregnated layer. Oper Dent 19:59-64.

Sano H, Takatsu T, Ciucchi B, Horner JA, Pashley DH (1994b). Nanoleakage: leakage within the hybrid layer (abstract). J Dent Res 73:276.

Suzuki M, Gwinnett AJ, Jordan RE (1989). Relationship between composite resin and dentin treated with bonding agents. $J$ Am Dent Assoc 118:75-77.

Swift EJ (1991). Microleakage of dentin adhesive systems. J Esthet Dent 3:91-94.

Tagami J, Nakajima M, Shono T, Takatsu T, Hosoda H (1993). Effect of aging on dentin bonding. Am J Dent 6:145-147.

Tay FR, Gwinnett AJ, Pang KM, Wei SHY (1994a). Structural evidence of a sealed tissue interface with a total etch wet bonding technique in vivo. J Dent Res 73:629-636.

Tay FR, Pang KM, Gwinnett AJ, Wei SHY (1994b). A method for microleakage evaluation along the dentin/restorative interface. Am J Dent 8:105-108.

Taylor MJ, Lynch E (1992). Microleakage. J Dent 20:3-10.

Tjan AHL, Bergh BH, Lidner CL (1992). Effect of various incremental techniques on the marginal adaptation of class II composite resin restorations. J Prosthet Dent 67:62-66.

Torstenson B, Brännström M (1988). Contraction gap under composite resin restorations: effect of hygroscopic expansion and thermal stress. Oper Dent 13:24-31.

Triadan H (1987). When is microleakage a real clinical problem? Oper Dent 12:153-157.

Van Meerbeek B, Inokoshi S, Braem M, Lambrechts P, Vanherle G (1992). Morphological aspects of the resin-dentin interdiffusion zone with different dentin adhesive systems. J Dent Res 71:1530-1540.

Van Meerbeek B, Dhem A, Goret-Nicaise M, Braem M, Lambrechts P, Vanherle G (1993). Comparative SEM and TEM examination of the ultrastructure of the resin-dentin interdiffusion zone. J Dent Res 72:495-501.

Watt IM (1985). The principles and practice of electron microscopy. Cambridge, UK: Cambridge University Press, pp. 64-70.

Wieczkowski G Jr, Yu XY, Davis EL, Joynt RB (1992). Microleakage in various dentin bonding agent/composite resin systems. Oper Dent (Suppl 5):62-67.

Wu W, Cobb EN, Dermann K, Rupp NW (1983). Detecting marginal leakage of dental composite restorations. J Biomed Mater Res 17:37-43. 